\title{
Intraoperative MR and Synthetic Imaging
}

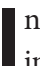

the past 2 decades, intraoperative MRI (ioMRI) has been implemented in surgery for pituitary tumors, low-grade gliomas, ${ }^{1}$ epilepsy, and deep brain stimulation. In neuro-oncological surgery, the adjunct of ioMRI improves the extent of resection, which eventually results in improved overall and progression-free survival. $^{2}$ However, despite encouraging results, there are no specific sequences dedicated to ioMRI to our knowledge.

In the neuroradiologist's point of view, one of the main difficulties is to find the most useful and time-effective sequence to guide the surgeon in determining the existence of residual tumor tissue. Often, multiple series before and after the administration of a contrast agent are used. In our opinion, this time-consuming step is not compatible with a surgical context in which time for imaging is limited. This restricts the neuroradiologist, especially in complex cases. One potential solution might reside in the use of so-called synthetic imaging (SyntheticMR; SyntheticMR, Linköping, Sweden), which allows us to obtain 1) different types of contrast, and 2) quantitative information, such as T1 and T2 mapping of the tissues. 3,4

The use of SyntheticMR in low-grade tumors could represent a real shift of paradigm. Due to edema, air, blood, and immediate postoperative changes, these lesions do not allow a clear delimitation of the tumor border solely by visual analysis, and remaining tumor may thus be missed. Having multiple contrasts in a single sequence by using SyntheticMR may aid the interpretation, in a reduced acquisition time $(7 \mathrm{~min})$. The quantitative values may also be of great interest; indeed, synthetic imaging provides several types of cartography, such as cerebral cortex, CSF, white matter, and other tissues, as shown in Fig 1. Quantitative values of $\mathrm{T} 1, \mathrm{~T} 2$, and proton density of the tissues are also available. It has already been shown that relaxation rates can be used to grade gliomas, ${ }^{5}$ but, at that time, there were no sequences with short acquisition times. Recently, this finding was confirmed with an MR fingerprinting technique. ${ }^{6}$ The latter sequence is under development and therefore, not fully available, contrary to SyntheticMR. In Fig 2, we show that T2 measured with SyntheticMR in the case of a World Health Organization grade I left occipital meningioma is different between the lesion and the cerebral cortex. In our

Please address correspondence to Maria Isabel Vargas, MD, Division of Diagnostic and Interventional Neuroradiology, Geneva University Hospitals, Rue GabriellePerret-Gentil 4, 1211 Genève 14, Switzerland; e-mail: maria.i.vargas@hcuge.ch; @maiva96

http://dx.doi.org/10.3174/ajnr.A6373 opinion, this principle could be used to help delineate remnant tumor tissue also in low-grade tumors.

We, therefore, think that SyntheticMR could be beneficial for ioMRI by providing multiple contrasts and relaxation-rate quantification to give a reliable interpretation of the presence of residual tumor, thus improving patient care.

Disclosures: None.

\section{ACKNOWLEDGEMENT}

The authors thank IMRIS. The SyntheticMR sequence (MDME) was provided by Karolinska University Hospital, developed in collaboration with Synthetic MR AB and Frederik Testud, Siemens Healthcare AB, Sweden.

\section{REFERENCES}

1. Coburger J, Merkel A, Scherer M, et al. Low-grade glioma surgery in intraoperative magnetic resonance imaging: results of a multicenter retrospective assessment of the German study group for intraoperative magnetic resonance imaging. Neurosurgery 2016;78:775-86 CrossRef Medline

2. Senft $C$, Bink A, Franz K, et al. Intraoperative MRI guidance and extent of resection in glioma surgery: a randomised, controlled trial. Lancet Oncol 2011;12:997-1003 CrossRef Medline

3. Warntjes JBM , Leinhard OD, West J, et al. Rapid magnetic resonance quantification on the brain: Optimization for clinical usage. Magn. Reson. Med. 2008;60:320-9 CrossRef Medline

4. Granberg T, Uppman M, Hashim F, et al. Clinical Feasibility of Synthetic MRI in Multiple Sclerosis: A Diagnostic and Volumetric Validation Study. Am. J. Neuroradiol. 2016;37:1023-9 CrossRef Medline

5. Newman S, Haughton VM, Yetkin Z, et al. TI, T2 and proton density measurements in the grading of cerebral gliomas. Eur Radiol 1993;49-52

6. Badve C, Yu A, Dastmalchian S, et al. MR fingerprinting of adult brain tumors: initial experience. AJNR Am J Neuroradiol 2017;38: 492-99 CrossRef Medline 


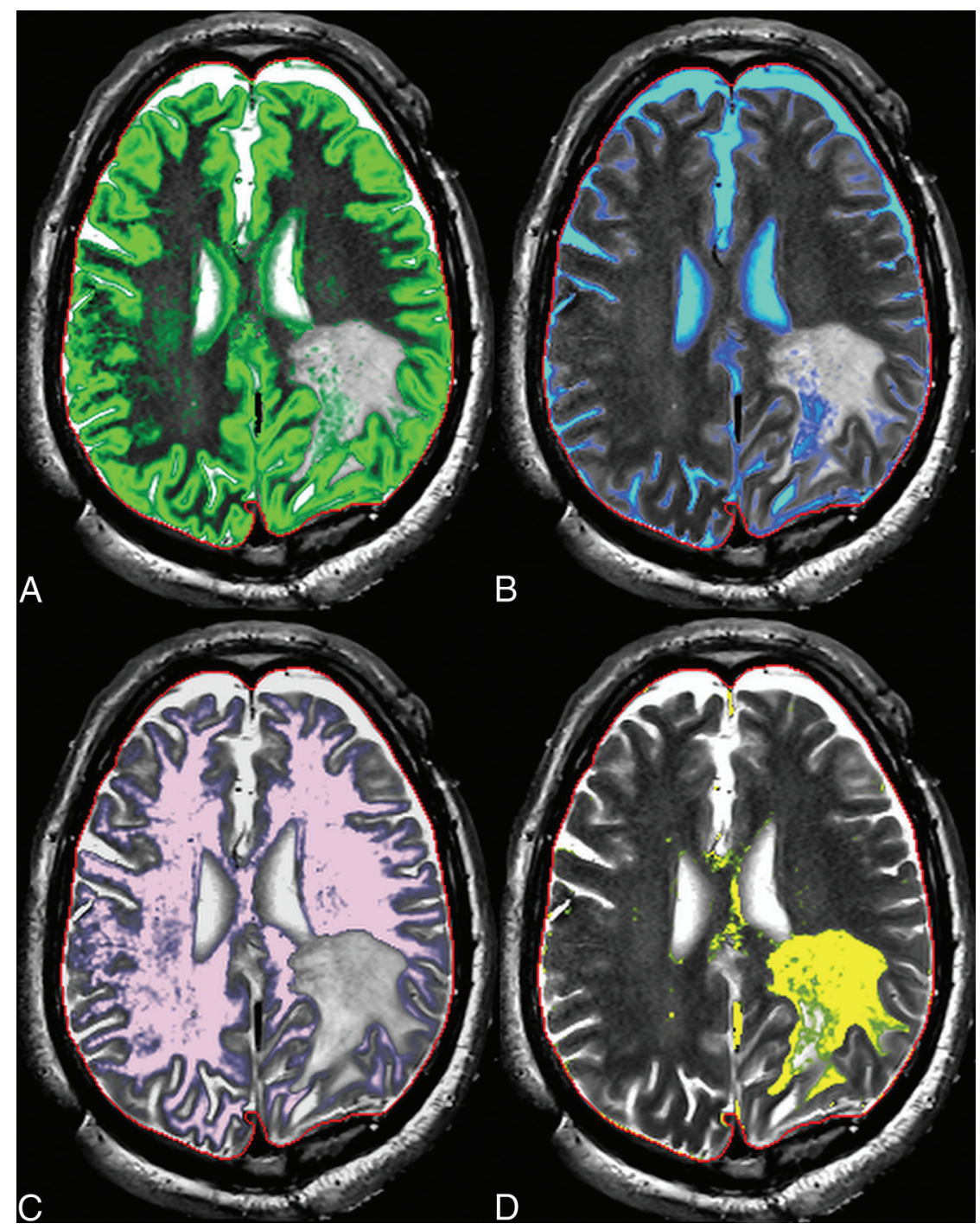

FIG 1. Intraoperative Synthetic MR cartographies illustrating gray matter (green in A), CSF (blue in B), white matter (pink in C), and edema (yellow in $D)$. 


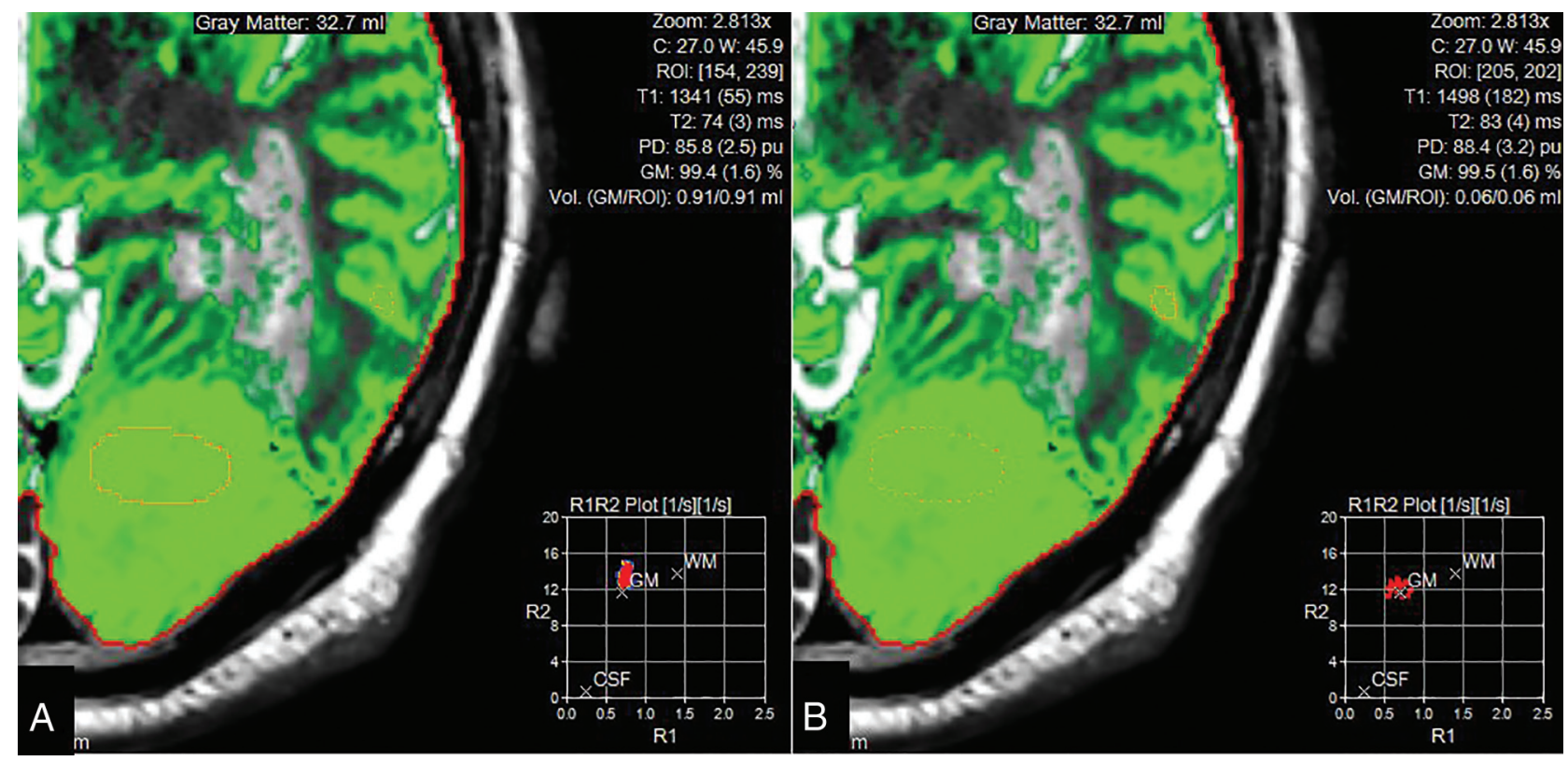

FIG 2. Region of interest in the tumor $(A)$, showing decreased $T 2$ values in rapport to gray matter $(B)$ and, on the contrary, $T 1$ values similar to the gray matter. 